\title{
Moderniser la contre-révolution : le carlisme pendant le Sexennat Démocratique
}

Modernizar la contrarrevolución : el carlismo durante el Sexenio Democrático Modernizing the Counter-revolution: the carlism during the Six Democratic Years

\section{Alexandre Dupont}

\section{OpenEdition}

\section{Journals}

Édition électronique

URL : http://journals.openedition.org/bhce/2027

DOI : $10.4000 /$ bhce.2027

ISSN : 1968-3723

Éditeur

Presses Universitaires de Provence

Référence électronique

Alexandre Dupont, « Moderniser la contre-révolution : le carlisme pendant le Sexennat Démocratique », Bulletin d'Histoire Contemporaine de l'Espagne [En ligne], 55 | 2020, mis en ligne le 01 septembre 2020, consulté le 28 novembre 2020. URL : http://journals.openedition.org/bhce/2027 ; DOI : https://doi.org/ 10.4000/bhce.2027

Ce document a été généré automatiquement le 28 novembre 2020.

Bulletin d'histoire contemporaine de l'Espagne 


\section{Moderniser la contre-révolution : le carlisme pendant le Sexennat Démocratique}

Modernizar la contrarrevolución : el carlismo durante el Sexenio Democrático

Modernizing the Counter-revolution: the carlism during the Six Democratic

Years

Alexandre Dupont

“Le Duc de Madrid sait que le triomphe de la justice et de la liberté chrétienne ne se trouve pas au fond des urnes électorales ; le Duc de Madrid sait que ces lamentables luttes, tout comme la recherche fébrile et incessante de la Presse périodique, tout comme tous les moyens de destruction employés avec tant de bonne fortune par le libéralisme, inspirent une profonde aversion au grand parti national ; il sait, enfin, et applaudit et approuve cela de tout cœur, que les hommes d'esprit viril et d'un haut courage, préfèrent plutôt risquer héroïquement leur vie et rafraîchir de leur propre sang les lauriers de Herrera et des Peñas de San Fausto, que de s'abaisser à faire échouer et briser les ruses et les misérables intrigues par lesquelles les Gouvernements libéraux cherchent à prolonger pour quelques heures leur insupportable tyrannie, rendant plus odieuses les luttes électorales, et les transformant en farces, ridicules ou sanglantes $\star^{1}$. 
1 C'est par ces considérations paradoxales que la Junte Centrale CatholiqueMonarchique, formée par Cándido Nocedal, Antonio de Vildósola, Manuel Tamayo et Vicente de la Hoz, justifiait la décision du prétendant don Carlos de Bourbon et d'Autriche-Este de participer aux élections législatives d'avril 1872. Les pages suivantes expliquaient en effet au peuple carliste que l'abandon de la lutte électorale équivaudrait à un suicide du parti et que les carlistes devaient recourir à tous les moyens à leur disposition pour préparer leur reprise du pouvoir. Le manifeste était daté du 8 mars. Un mois plus tard, alors que les carlistes avaient connu un recul lors des élections du 3 avril, le prétendant donna l'ordre à la quarantaine de députés du parti de se retirer du Congrès et appela ses partisans à prendre les armes pour reconquérir le pouvoir par la force. Le 14 avril 1872 marqua le début de la Seconde guerre carliste.

2 L'historiographie a traditionnellement considéré que l'année 1872 marquait un tournant dans l'histoire du carlisme pendant la période du Sexennat démocratique : à la participation démocratique au jeu politique ouvert par la Révolution de 1868 aurait succédé la prise d'armes par laquelle le carlisme aurait renoué avec ses moyens d'action des années 1830 et 1840 . Les explications données par les historiens successifs du carlisme à ce changement de stratégie permettent dès l'abord de retracer à grands traits l'historiographie du carlisme au cours du Sexennat. Les historiens pro-carlistes pointent en général la déloyauté des libéraux au pouvoir, incapables de respecter les règles d'un jeu démocratique qu'ils avaient pourtant eux-mêmes instauré, ainsi que le trucage des élections de 1872 pour expliquer le soulèvement carliste. C'est la position défendue notamment par Melchor Ferrer : les carlistes auraient été forcés de prendre les armes pour contrer un libéralisme anti-démocratique et anticatholique ${ }^{2}$.

3 De façon générale, les historiens non-carlistes mettent l'accent sur les dissensions qui existaient dans le camp carliste entre partisans de l'action légale et partisans de l'action armée, ces derniers emportant la décision en 1872. C'est le constat fait dès la fin du XIX ${ }^{\mathrm{e}}$ siècle par Antonio Pirala, et qui n'a pas été remis en cause par la suite ${ }^{3}$. Les interprétations données à ces tensions ont en revanche évolué.

Dans un premier temps, on a voulu y voir les querelles entre carlistes historiques et néo-catholiques ralliés dans la deuxième moitié des années 1860, plus enclins à accepter le parlementarisme. Les études d'histoire économique et sociale produites à partir des années 1970, parfois depuis une perspective marxiste, ont mis en avant le mécontentement socio-économique existant dans les régions de forte implantation carliste et ont interprété la guerre comme le résultat de la pression des classes populaires carlistes en faveur d'un soulèvement armé. Enfin, les études d'histoire politique et culturelle qui se sont développées à partir des années 1990 ont vu dans la prise d'armes le résultat d'un processus de modernisation inachevée du carlisme, celuici ayant tenté de se transformer en un parti politique moderne avant que les contradictions internes à ce processus ne le poussent à reprendre la voie des armes, mettant provisoirement un terme à cette modernisation. Ces mêmes historiens ont aussi remis en avant les dimensions proprement culturelles de la mobilisation, en particulier autour de la question religieuse et de la remise en cause de la place du catholicisme dans l'État et la société espagnols.

5 On le voit, ces interprétations ne s'excluent pas entre elles et soulignent l'existence d'une historiographie riche sur cette étape du Sexennat, historiographie à laquelle l'auteur de ces lignes participe modestement depuis quelques années en soulignant les 
déterminants transnationaux de l'action des carlistes. En outre, l'étude du carlisme a bénéficié au cours des vingt dernières années des renouvellements historiographiques ayant trait à la contre-révolution européenne en général. C'est à la lumière de ces renouvellements que le présent article se propose d'actualiser le récit toujours largement valable proposé par Jordi Canal en 2000 dans sa synthèse de référence sur le carlisme ${ }^{4}$. Sans prétendre à l'exhaustivité ni à l'originalité, le propos développé dans les pages qui suivent prétend ouvrir des perspectives de recherches sur le carlisme et sa place dans une étape-clé de l'histoire de l'Espagne.

On nous permettra avant cela de faire un bref état des lieux des sources disponibles sur le carlisme. Outre les très nombreuses archives réparties dans les centres du Pays basque, de la Navarre, de la Catalogne, de la communauté valencienne et de l'Aragon, les historiens qui souhaiteraient travailler sur le carlisme pendant le Sexennat peuvent s'appuyer sur trois fonds essentiels. Pratiqués de longue date par les historiens du carlisme, les papiers récoltés par l'historien Antonio Pirala auprès des chefs carlistes à la fin de la guerre de 1872-1876 constituent un matériel de première main assez inestimable dans le paysage des archives sur la contre-révolution en Europe ${ }^{5}$. À ce fonds, qui reste encore largement à exploiter, se sont ajoutés au cours des dernières décennies deux autres ensembles archivistiques de grande importance. En 2002, la famille de Bourbon-Parme a confié à l'Archivo Histórico Nacional de Madrid les documents des dynastes carlistes en sa possession ${ }^{6}$. Pour la période du Sexennat, il s'agit notamment de la correspondance d'Alfonso de Bourbon et d'Autriche-Este, frère du prétendant don Carlos, et de sa femme Marie des Neiges de Bragance, sur laquelle une thèse de doctorat est actuellement en préparation?. Transférés à l'Archivo General de la Universidad de Navarra, où leur classement a été entièrement repris, les papiers de l'historien carliste Melchor Ferrer constituent également un matériau précieux pour les historiens du carlisme ${ }^{8}$.

7 À ces fonds, il faudrait ajouter les archives militaires espagnoles, mais aussi les archives diplomatiques, espagnoles et françaises au premier chef. Surtout, l'histoire du carlisme au cours du Sexennat peut s'appuyer sur le grand nombre de sources imprimées publiées par ou sur les carlistes au cours de cette période. Ce sont ces sources qui ont été les plus utilisées par les chercheurs, notamment dans les travaux sur l'évolution de l'idéologie carliste, sur les phénomènes de médiatisation et de popularisation de la cause etc. La presse, en particulier, constitue un massif archivistique fondamental, en raison de l'explosion du nombre de journaux, permise par la démocratisation qui a lieu lors du Sexennat. À ce titre, et pour ne pas y revenir par la suite, il reste encore beaucoup de travail à faire sur cette presse carliste, surtout celle qui apparaît un peu partout en province. De façon générale, ces sources imprimées offrent un regard précieux sur les processus à l'œuvre au sein du carlisme à cette époque. Un massif encore relativement peu utilisé, ou alors très ponctuellement, concerne enfin les ressources iconographiques et matérielles disponibles sur le carlisme : suivant en cela des évolutions historiographiques générales à l'œuvre, on peut espérer que les historiens se penchent sur ces productions, mises en valeur depuis quelques années par un certain nombre d'institutions culturelles, comme le Museo Zumalakarregi d'Ormaiztegi, le Museo del Carlismo d'Estella, le Musée basque et d'histoire de Bayonne ainsi que la Fundación Sancho el Sabio de Vitoria-Gasteiz. 


\section{La renaissance du carlisme}

8 La spectaculaire renaissance opérée par le carlisme dans les premières années du Sexennat Démocratique constitue un phénomène politique complexe qui doit être compris à plusieurs échelles. Au moment où éclata la Glorieuse Révolution de septembre 1868, qui mit fin au régime libéral-conservateur de la reine Isabelle II, la culture politique carliste n'était plus que l'ombre d'elle-même. Les années 1860 avaient été le théâtre d'une triple crise qui avait failli la mettre à bas définitivement. Une crise stratégique s'était ouverte dès 1860, lorsque la désastreuse expédition de San Carlos de la Rápita, qui s'était conclue par l'arrestation du prétendant Carlos Luis et de son frère, avait révélé l'impasse de la stratégie insurrectionnelle suivie depuis la défaite de 1840 et l'échec des mariages espagnols dans les années suivantes. À cette crise stratégique s'était rapidement ajouté une crise dynastique, lorsque les décès en quelques mois de Carlos Luis et de son frère Fernando en 1861 firent accéder à la prétendance ${ }^{9}$ le troisième frère, Juan, qui affirma bientôt ses convictions libérales et son ralliement au trône de sa cousine Isabelle.

Cette orientation ouvrit immédiatement une crise politique au sein du carlisme qui déstabilisa profondément la cohérence d'une culture politique fondée sur des valeurs contre-révolutionnaires partagées ${ }^{10}:$ prééminence du catholicisme comme système de valeurs et de l'Église comme institution sociale, centralité de la figure royale comme symbole de l'unité politique, institutionnelle et nationale, organisation socioéconomique inspirée de l'Ancien Régime reposant sur le concept de communauté et sur l'existence de particularismes locaux et régionaux ${ }^{11}$. L'impossibilité logique, aux yeux des contre-révolutionnaires, que constituait la défense du libéralisme par le prétendant carliste constitua une menace sérieuse. Il fallut l'intervention décisive dans le débat de la princesse de Beira, femme du premier don Carlos, pour résoudre la crise : dans sa Lettre aux Espagnols de 1864, elle affirma que le respect du dogme politique prévalait sur la légitimité dynastique, et que si Juan refusait de se conformer aux idées du carlisme, le droit de prétendre au trône d'Espagne passerait à son fils Carlos ${ }^{12}$. Il n'y avait là rien de nouveau : sous les apparences d'une querelle dynastique entre don Carlos et sa nièce Isabelle, c'était bien un combat politique qui était au cœur de la Première guerre carliste, comme ce serait le cas entre 1872 et 1876 . Toutefois, en l'exprimant de façon aussi explicite, la princesse de Beira actait le fait que le carlisme était bien une culture politique comme les autres dans l'Europe du XIX ${ }^{e}$ siècle, un regroupement de gens qui défendaient un corpus d'idées et un projet commun pour le futur de leur pays ${ }^{13}$.

Le retournement de situation se produisit pour le carlisme dans la deuxième moitié des années 1860 , sous l'effet de plusieurs processus. D'abord, la crise interne qui l'avait miné depuis le début de la décennie finit par se résoudre lorsque don Juan fut contraint d'abdiquer en faveur de son fils, qui devint prétendant en 1868 et prit le titre de duc de Madrid. Don Carlos de Bourbon et d'Autriche-Este, né en 1848, avait été élevé dans une stricte orthodoxie contre-révolutionnaire par sa mère Béatrice de Modène, ce qui lui valut le surnom, donné par ses partisans et moqué par ses adversaires, d'enfant sans tâche (el niño terso). Âgé de vingt ans, il était fermement décidé à opérer une réorganisation du carlisme. Si le romancier Theo Aronson a pu dire à son propos que “des générations de jars avaient donné naissance à un cygne ${ }^{14}$, si les qualités politiques du prince, dont la biographie reste à écrire, étaient réelles, on ne saurait en 
rester à cette approche classique de la contre-révolution, par la figure des princes et par le haut ${ }^{15}$.

11 La renaissance du carlisme s'inscrivit aussi dans un mouvement européen voire mondial. Depuis la fin des années 1850, plusieurs processus étaient à l'œuvre qui avaient commencé à miner l'édifice politique précaire sorti des contre-révolutions de 1849. En particulier, la péninsule italienne était devenue dans les années 1860 un hautlieu de l'affrontement entre révolution et contre-révolution, entre le libéralisme et le droit des peuples d'une part et la défense des Anciens Régimes et de la religion d'autre part. Ce contexte influença grandement les carlistes, qui furent à la pointe de la mobilisation en faveur du roi des Deux-Siciles François II, détrôné par l'unification italienne ${ }^{16}$. Surtout, les menaces que le processus unitaire fit peser sur la souveraineté temporelle des papes tout au long de la décennie provoquèrent une intense mobilisation des catholiques face à ce qu'ils interprétaient comme une nouvelle attaque diabolique contre l'Église ${ }^{17}$. L'attitude des catholiques espagnols n'a pas encore été étudiée dans sa globalité, mais il est certain que la situation italienne détermina la rupture du secteur néo-catholique avec la reine Isabelle II, que l'échiquier diplomatique avait contraint à reconnaître le royaume d'Italie en 1865 , et son ralliement au carlisme $^{18}$. Des Espagnols s'engagèrent par ailleurs dans le corps international des Zouaves Pontificaux, et notamment le prince Alfonso de Bourbon et d'Autriche-Este, frère cadet de don Carlos ${ }^{19}$.

12 On comprend mieux dans quel état d'esprit les catholiques espagnols accueillirent la Révolution de Septembre et l'installation d'un régime démocratique et libéral. Quelle que fût leur satisfaction de voir s'effondrer un régime honni, qui avait selon eux provoqué sa perte en pactisant avec la Révolution, les mesures libérales en matière religieuse qui furent prises dès 1868 les incitèrent à interpréter cette révolution comme une nouvelle avancée de Satan en Europe. En particulier, le débat sur la liberté de culte aux Cortès constituantes de 1869 provoqua une mobilisation importante des catholiques contre cette disposition. On commence tout juste à étudier cette mobilisation ${ }^{20}$, mais il est indéniable qu'elle participa à la constitution de ce que Jordi Canal avait élégamment qualifié d"' amalgame contre-révolutionnaire ${ }^{21}$. La situation révolutionnaire et la peur qu'elle provoqua chez une partie de la population espagnole permirent au carlisme d'apparaitre comme le rempart de l'ordre et de la religion argument qu'il ne manqua pas d'utiliser au cours de ces années, résumant la situation à la manière du chanoine Manterola: "don Carlos ou le pétrole $»^{22}$. Autrement dit, le carlisme agrégea autour de lui tout ce que l'Espagne comptait d'esprits conservateurs et catholiques effrayés par la révolution, et qui considéraient qu'à tout prendre, mieux valait une restauration de don Carlos qu'une révolution sociale.

13 Dans ce processus, l'appareil carliste ne fut pas passif, au contraire. Paradoxalement, malgré son opposition résolue aux mesures décidées par le gouvernement provisoire puis par les gouvernements qui se succédèrent au pouvoir de 1869 à 1872, les responsables carlistes firent le choix dès le mois d'octobre 1868 d'accepter les conditions de la vie politique légale. J'ai eu l'occasion d'examiner les raisons de ce choix dans plusieurs articles ${ }^{23}$. La plus importante correspond sans doute à une transformation observable dans l'ensemble des mouvements contre-révolutionnaires européens $^{24}$, qui les vit passer d'un refus de la politique et d'une conception élitaire de la société à une intégration paradoxale du peuple comme instance de légitimation dans le corpus idéologique et à la valorisation du combat politique légal. Face à leur 
marginalisation dans les appareils d'État, les royalistes de tous les pays virent dans les classes populaires un réservoir d'électeurs qui devaient enfin permettre leur retour au pouvoir et chasser des libéraux forcément minoritaires et illégitimes. L'influence du catholicisme intransigeant promu par Pie IX, qui s'efforça de reconquérir les foules par des pratiques de masse comme les pèlerinages ou les processions ${ }^{25}$, ne fut pas pour rien dans ce processus de “ démocratisation » de la contre-révolution, et en l'occurrence du carlisme.

Ayant fait ce choix, les responsables carlistes décidèrent aussi de réorganiser l'appareil du parti. Parler de parti jusqu'en 1868 n'a d'ailleurs guère de sens, et constitue une facilité de langage. Jusqu'alors, le carlisme était une culture politique au sens propre du terme, une communauté de valeurs, de symboles, de sentiments et d'expériences qui en rassemblait les membres. À compter de 1868, avec la création de la Communion catholique-monarchiste, il est possible d'évoquer un parti politique, même si le terme ne recouvrait pas alors l'acception qu'il prendrait en Espagne dans les dernières décennies du XIX ${ }^{\mathrm{e}}$ siècle $^{26}$.

En tout cas, dans les premières années du Sexennat, la Communion catholiquemonarchiste devint une véritable structure d'organisation et de mobilisation du peuple carliste, un proto-parti qui se lança dans la vie électorale démocratique introduite pour la première fois en Espagne par la Constitution de 1869. Le carlisme disposait d'un appareil cohérent, hiérarchisé et organisé qui aspirait à incarner dans le champ de la politique légale la culture politique qui existait depuis les années 1820 et les ultras du règne de Ferdinand VII. De ce point de vue, l'apport des néo-catholiques fut sans doute décisif. Né dans les cercles du pouvoir isabellin, ce groupe de journalistes, de parlementaires, d'écrivains, de juristes et de penseurs apporta au carlisme son expérience en termes de pratiques politiques légales. Le phénomène fut particulièrement visible dans les travées des Cortès, où des hommes comme Cándido Nocedal s'imposèrent comme les orateurs du parti, mais aussi dans l'évolution du bagage idéologique carliste, qui connut une réelle transformation sous la plume d'Antonio Aparisi y Guijarro ${ }^{27}$.

Cette réorganisation du carlisme en un parti structuré et cohérent fut d'autant plus intéressante qu'elle eut lieu à plusieurs échelles, et qu'on ne saurait l'expliquer sans examiner ses dimensions transnationales ${ }^{28}$. D'abord à Londres, ensuite à Paris et enfin à Vevey au bord du lac Léman, don Carlos réunit autour de lui un grand nombre de chefs carlistes qui travaillèrent depuis l'exil à cette reconstruction de la Communion. Le carlisme se transforma en l'un des principaux acteurs de la scène politique espagnole grâce à l'œuvre menée depuis l'étranger par ses responsables. Cela n'avait du reste rien d'original ni de nouveau dans l'histoire politique de l'Espagne au XIX ${ }^{e}$ siècle. Pour autant, la place des influences internationales et des circulations dans cette réorganisation sont encore mal connues: l'analyse détaillée des comptes rendus de séances du conseil royal réuni à Paris autour de don Carlos en 1869 apporterait sans doute de nombreux éclairages à cet égard ${ }^{29}$. Quoi qu'il en soit, cette exopolitie ${ }^{30}$ des responsables carlistes ne déboucha pas sur un parti hors-sol et déconnecté des réalités du pays. Au contraire, un soin particulier fut apporté au maillage du territoire par des structures locales de politisation. La mobilisation des classes populaires catholiques et carlistes fut en effet une dimension fondamentale de l'histoire du carlisme pendant le Sexennat. 


\section{Politisation et mobilisation du " peuple carliste "}

17 Ce récit institutionnel et par le haut de la renaissance du carlisme prévaut encore largement. Y compris dans les quelques analyses dont on dispose sur la politisation populaire carliste, c'est un modèle top-down, pour reprendre les distinctions classiques $^{31}$, qui domine généralement. Les historiens du carlisme ont en particulier mis en avant le rôle joué par la presse dans la politisation des catégories populaires. De façon générale, le Sexennat fut un moment crucial de politisation par ce biais: le rétablissement de la liberté de la presse par le gouvernement provisoire dès les lendemains de la révolution provoqua une véritable explosion du nombre de titres. De ce point de vue, les carlistes ne furent pas en reste, qui démontrèrent leur capacité à s'emparer de pratiques culturelles modernes pour les utiliser à leur avantage. Que ce soit à Madrid ou, surtout, en province, les journaux carlistes se multiplièrent et constituèrent un relais essentiel de la propagande du parti. On connaît bien désormais La Esperanza, figure de proue de ce dispositif médiatique, grâce à l'étude d'Esperanza Carpizo Bergareche ${ }^{32}$. On dispose également d'études ponctuelles sur d'autres titres, mais comme je l'ai dit plus haut, le travail reste encore largement à faire sur ce vecteur essentiel de la politisation au XIX $x^{e}$ siècle.

De façon plus générale, tout le matériel mis à profit par la Communion catholiquemonarchiste pour gagner de nouveaux électeurs dans le cadre de la stratégie légaliste demanderait à être examiné en profondeur. On sait que les carlistes tirèrent parti de tous types d'imprimés à leur disposition, de la chanson au poème, en passant par la lettre ouverte et la proclamation. On sait aussi qu'ils firent grand usage des images, y compris de la photographie et plus spécifiquement des portraits du prince, qui acquit dès lors une médiatisation toute différente de celle de son oncle ou de son grand-père ${ }^{33}$. Ces éléments rappellent combien le carlisme, au temps du Sexennat, ne constituait pas une culture politique recluse dans le passé et refusant à tout crin la modernité, mais bien un mouvement dynamique, capable de s'approprier les techniques politiques modernes pour les adapter à son corpus idéologique.

19 À l'inverse, les responsables de la Communion surent aussi investir les formes particulières de la sociabilité contre-révolutionnaire pour faire progresser leurs idées. La messe fut ainsi un lieu essentiel de la politisation, et la place des ecclésiastiques dans la mobilisation des classes populaires carlistes est un chantier encore en jachère - il s'agirait notamment de comprendre les raisons pour lesquelles tant de prêtres se trouvèrent à la tête de bandes armées lors du soulèvement de $1872^{34}$. Les structures partisanes de la Communion ressuscitaient quant à elles les hiérarchies sociales fondées sur la domination des élites traditionnelles au niveau local: ce furent souvent des notables qui furent mis à la tête de telle ou telle cellule de la Communion ${ }^{35}$.

Si des structures verticales de politisation furent mises en place, elles coexistèrent avec des processus de politisation par en bas, ce que les historiens désignent par le modèle bottom-up, ou encore par politisation ordinaire. Le carlisme disposait en effet, depuis le début du siècle, d'une audience populaire incontestable. À côté de la petite noblesse et d'une part conséquente du clergé, les idées contre-révolutionnaires avaient séduit un public composé majoritairement de paysans et d'artisans, en particulier dans le nord du pays, et plus précisément encore dans les provinces basques, dans la Catalogne intérieure et aux confins de l'Aragon et du pays valencien, dans le Maeztrazgo ${ }^{36}$. 
21 Cette géographie sommaire n'épuise pas l'ensemble des zones d'implantation du carlisme, mais dans ces régions, cette culture politique devint un facteur d'organisation sociale, un ferment de solidarité des communautés locales, notamment dans les campagnes. Le carlisme s'appuyait en effet sur un clivage ville/campagne bien visible dans l'espace basque, et qui constitua une difficulté permanente dans ses tentatives militaires. Cet enracinement local est étroitement lié aux phénomènes de politisation informelle. Dans ces espaces, la politisation - ou la repolitisation - des premières années du Sexennat venait seulement se couler dans des structures sociales et collectives déjà fortement marquées par l'empreinte carliste lors des décennies précédentes. La construction d'identités collectives locales autour de la défense du catholicisme, de la figure du prétendant, des fueros ou encore de l'expérience de la lutte armée fut aussi décisive que le contexte révolutionnaire ou l'action des responsables carlistes $^{37}$. C'est notamment ce qu'ont montré des historiens comme Mikel Urquijo pour le Pays basque ${ }^{38}$ ou Lluis Ferran Toledano González pour la Catalogne ${ }^{39}$, qui ont bien mis en avant la place des tensions religieuses et politiques internes aux communautés villageoises dans la dynamique qui mena au conflit de 1872-1876.

Un apport stimulant sur cette politisation ordinaire des classes populaires carlistes est dû aux travaux d'Enriqueta Sesmero, qui s'est penchée sur la place des déterminants socio-économiques dans la mobilisation populaire au Pays basque ${ }^{40}$. Elle a ainsi montré que la fragilisation économique liée à l'installation du libéralisme en Espagne ainsi qu'à la crise de 1865-1866 avait joué un rôle non négligeable dans la renaissance d'une conflictualité socio-politique importante dans ces zones du nord du pays où existait une petite paysannerie et un milieu artisan attachés aux structures socio-économiques d'Ancien Régime - autre façon de désigner les fueros. Cette approche mériterait d'être reprise à nouveaux frais, à une échelle plus large tant géographiquement que temporellement, dans la mesure où les effets concrets de la révolution libérale sur le plan social restent trop mal connus ${ }^{41}$.

23 Depuis quelques décennies, l'approche culturelle du politique a été privilégiée par les historiens, qui souhaitaient à raison sortir d'une conception assez mécaniste de la politisation, qui aurait vu ce processus comme une réponse automatique à la condition économique des acteurs et aux structures sociales qui s'imposaient à eux ${ }^{42}$. Cette vision quelque peu simpliste, qui a pu être celle d'une partie de l'école marxiste, n'enlève pourtant pas sa pertinence à une approche par l'économie et le social de la politisation. Il est en effet possible de réintégrer ces dimensions dans un questionnement plus large sur la vision du monde et les espaces du politique de ces catégories populaires ${ }^{43}$. À ce titre, on connaît encore trop peu les effets dans les communautés locales des désamortissements successifs de 1836 et 1855 . Ce dernier, qui visait les biens communs détenus par les communes, a sans nul doute contribué à déstabiliser les équilibres socio-économiques de ces communautés paysannes ${ }^{44}$. Dès lors, une réflexion à nouveaux frais sur le vieux débat des fueros pourrait être menée à partir d'une histoire économique qui intègrerait également des questionnements environnementaux.

Plus largement, il s'agirait de reconsidérer ces processus de politisation populaire pendant le Sexennat en sortant du vieux débat entre politisation par le haut et politisation par le bas. Dans le cas de la contre-révolution, et ici du carlisme, ce débat visait à comprendre comment des individus issus des catégories populaires avaient pu faire un choix apparemment contraire à leurs intérêts objectifs, le postulat de départ étant que les classes populaires n'avaient rien à gagner à défendre un système d'Ancien 
Régime. Les réponses proposées jusqu'à présent sont à la fois valables et convaincantes, en particulier celles qui ont mis en valeur l'importance des déterminants culturels, la religion tout spécialement. Pour autant, le temps semble venu de faire un pas en avant, et de reconsidérer cette politisation populaire à l'aune de son originalité. Plutôt que d'essayer de rabattre la pensée politique des acteurs populaires du carlisme sur celle des élites, je crois que l'enjeu, comme j'ai essayé de le montrer dans plusieurs travaux ${ }^{45}$, est de prendre au sérieux la vision du monde des classes populaires, leur outillage mental pour reprendre un vocabulaire daté, dans son originalité et dans sa "rationalité spécifique $»^{46}$.

L'hypothèse que je défends, pour le dire d'un mot, est que la culture politique des acteurs populaires du carlisme n'est pas la même que celle des élites carlistes, autrement dit qu'il existe bel et bien une distinction entre ce que François Lebrun et Roger Dupuy avaient appelé la contre-révolution et l'anti-révolution ${ }^{47}$. Néanmoins, mon analyse de la politisation populaire carliste pendant le Sexennat diverge épistémologiquement sur deux points. D'une part, je ne pense pas qu'il y ait d'un côté une pensée politique cohérente et structurée - la contre-révolution - et de l'autre un rejet instinctif du libéralisme politique et économique - l'anti-révolution. Je crois au contraire qu'il y a là deux visions du monde présentant des points de convergence et des points de divergence, mais qui ont chacune leur cohérence.

D'autre part, il me semble que l'enjeu fondamental des recherches à venir sur le carlisme porte sur l'articulation de ces deux visions du monde. Contre-révolution élitaire et contre-révolution populaire, malgré leurs dissemblances, ont fonctionné en syntonie au point que la distinction entre les deux a pu s'effacer. Dès lors, cela suppose deux séries de questionnements: quels sont les vecteurs de dialogue et de mise en relation entre ces deux langages contre-révolutionnaires, en particulier s'agissant des acteurs (et il faudrait mobiliser ici le concept de go-between) ? comment ces deux niveaux, ces deux espaces, s'influencent-ils mutuellement, à différents niveaux ? Si les travaux historiques existants ont bien montré que les élites avaient contribué à la politisation des classes populaires, on ne s'est jamais penché sur le phénomène inverse, sur la façon dont les classes populaires avaient pu influencer les élites et leurs idées, imposer leurs préoccupations, modifier le discours politique dominant en fonction de leurs propres revendications.

On le voit à la lecture de ce qui précède, le chantier est en jachère. Il y a là un immense pan de l'histoire du carlisme durant le Sexennat qui nous reste encore largement inconnu et qui mériterait qu'on y travaille. Plus largement, ces questionnements, à mon sens, pourraient amener à une nouvelle compréhension des mouvements contrerévolutionnaires en Europe au xix ${ }^{e}$ siècle. Mais pour se limiter au Sexennat, deux éléments déjà étudiés plaident en faveur d'une telle vision dynamique de la politisation carliste. Dès les premières années, les élites carlistes repensèrent à nouveaux frais la question des fueros et en firent un élément définitoire de l'idéologie beaucoup plus qu'elle ne l'était avant. On peut raisonnablement supposer que ce furent les revendications économiques et sociales de la base qui conduisirent les cercles dirigeants de la Communion à opérer cette réorientation.

Enriqueta Sesmero a aussi montré que les classes populaires avaient joué un rôle majeur dans la montée de la conflictualité qui déboucha sur le conflit de 1872. À l'en croire, plus que les dissensions entre vieux généraux carlistes et parlementaires néocatholiques, entre partisans de la voie insurrectionnelle et défenseurs de la voie 
politique, ce fut la volonté des acteurs populaires de reprendre le chemin des montagnes qui tint une place décisive dans le déclenchement de la Seconde guerre carliste $^{48}$. Don Carlos finit par faire sienne la volonté d'en découdre de ses soutiens populaires. Il y a là encore des recherches à mener pour confirmer ou infirmer cette hypothèse. Plusieurs sources de l'époque font en tout cas voir la perplexité et l'inquiétude des dirigeants carlistes face à l'attitude belliqueuse leurs affidés dans les montagnes basques dans les mois précédant le conflit ${ }^{49}$.

\section{Dynamiques de la guerre civile}

La guerre est-elle un outil politique archaïque ? Le conflit déclenché par les carlistes en 1872 marqua-t-il le retour de cette famille politique à ses vieux démons, à la violence fratricide et à l'incapacité d'accepter les règles du jeu démocratique? À des degrés divers, c'est le constat qu'ont fait la plupart des historiens à propos de la Seconde guerre carliste ${ }^{50}$. Sur cet épisode pèsent en réalité les mânes de Norbert Elias et de Jürgen Habermas, qui constituent un impensé des historiens du politique au $\mathrm{xIX}^{\mathrm{e}}$ siècle ${ }^{51}$. L'émergence progressive au cours de ce siècle d'un espace normé et pacifique d'expression du politique, caractérisé par l'apparition de la démocratie libérale, aurait créé les conditions d'un abandon de la violence politique, celle-ci n'étant plus, dans ce contexte, ni nécessaire ni admissible. Cette pacification fondée sur une éthique de la discussion serait le volet politique du processus de civilisation, associant renforcement de l'État et autocontrôle des individus. C'est ce cadre mental qui conduit encore les historiens à considérer la violence politique, même s'il faut l'étudier, comme une anomalie ${ }^{52}$. Toutefois, on peut inverser la focale, prendre acte de l'omniprésence de cette violence dans l'Europe du XIX ${ }^{e}$ siècle et essayer d'en comprendre les logiques et les dynamiques en partant de l'idée que pour les acteurs eux-mêmes, la violence et la guerre étaient peut-être une façon de faire de la politique parmi d'autres.

30 Appliqué à la guerre carliste, un tel schéma interprétatif, qui n'est à ce stade qu'une hypothèse, permet de reconsidérer le basculement de 1872 et de cesser de le considérer comme une régression des carlistes vers des formes politiques primitives, voire comme une résurrection du "caïnisme" espagnol, qui verrait ce pays replonger périodiquement dans l'affrontement fratricide, au point que cela constituerait un de ses traits définitoires sur la scène européenne ${ }^{53}$. Jordi Canal avait déjà fait un sort à cette dernière interprétation en montrant que le soulèvement carliste de 1872 s'inscrivait dans une tendance européenne à la guerre civile au XIX ${ }^{e}$ siècle ${ }^{54}$. En particulier, les expériences italiennes de la décennie précédente, dans le Mezzogiorno comme à Rome, étaient venues rappeler que si particularisme de la guerre civile il y a, il faut l'entendre à l'échelle méditerranéenne et non péninsulaire. Si l'on accepte de considérer le recours à la guerre comme une option possible et non comme une macule honteuse, les dynamiques et les logiques du soulèvement doivent être reconsidérées, en particulier du point de vue de l'engagement des acteurs.

31 La guerre de 1872-1876 eut en effet plusieurs facettes. Les étapes du conflit sont connues $^{55}$. Le soulèvement dans les provinces basques en avril 1872 échoua lamentablement et donna lieu à la convention d'Amorebieta au début du mois de mai. Une guerre irrégulière se poursuivit toutefois en Catalogne jusqu'à la fin de l'année 1872 qui permit un nouveau soulèvement côté basque en décembre, ce qui marqua le début d'une véritable guerre civile, opposant deux armées. Notons toute l'importance 
de défataliser ${ }^{56}$ le récit de la guerre: elle ne différa des multiples tentatives de soulèvement qui avaient eu lieu dans les années précédentes que par le fait qu'elle réussit. Dès lors, penser l'histoire du carlisme pendant le Sexennat comme le passage d'une voie politique à une voie militaire conduit à masquer la conflictualité sociale qui se manifesta dès la Révolution de 1868 sur le terrain ${ }^{57}$.

Quoi qu'il en soit, les armées carlistes remportèrent rapidement un certain nombre de succès, que ce soit dans les provinces basques, où elles conquirent un territoire cohérent et relativement étendu, ou en Catalogne et dans le Centre où, sous la houlette de don Alfonso et de Francesc Savalls, elles ne contrôlèrent que quelques zones, qui incluaient des villes moyennes toutefois. Cette montée en puissance du carlisme militaire fut tout à la fois un catalyseur et le produit de l'instabilité qui régna à Madrid à partir de la fin de l'année 1872, et qui se prolongea au moins jusqu'au coup d'État qui mit au pouvoir le maréchal Serrano en décembre 1873. En 1874, la guerre atteignit son intensité maximale: les carlistes échouèrent une nouvelle fois à conquérir Bilbao au printemps malgré plusieurs mois de siège, mais leur victoire à Abarzuza à l'été contre les troupes républicaines leur laissa espérer une rupture du front qui leur eût permis de déferler sur la Castille. Si cette percée n'eut jamais lieu, la situation militaire n'avait clairement rien de réglé lorsque le général Martínez Campos réalisa son pronunciamiento en décembre 1874 pour mettre sur le trône Alphonse XII.

Selon un processus relativement bien connu, la restauration d'Alphonse XII sonna le glas des espoirs carlistes de victoire. La reconnaissance du nouveau pouvoir par le pape Pie IX et par le mythique chef carliste Ramón Cabrera, la séduction opérée sur les conservateurs par ce nouveau régime, les dissensions au sein du carlisme lui-même, furent autant d'éléments qui expliquèrent l'effondrement relativement rapide du carlisme au cours de l'année $1875^{58}$. La Catalogne fut la première à tomber à l'automne et les troupes alphonsistes se concentrèrent alors sur les provinces basques, qu'elles reconquirent aux mois de janvier et février 1876, poussant à l'exil près de vingt mille carlistes qui entrèrent en France et furent internés dans différentes villes du pays - une réalité dont on connaît à présent les grands traits, mais sur laquelle beaucoup reste à faire, même si l'auteur de ces lignes a proposé plusieurs contributions qui se penchaient sur les dimensions politiques de ces exils carlistes ${ }^{59}$.

L'autre apport auquel j'ai contribué au cours des dernières années à propos de la guerre carliste est son inscription dans des phénomènes de solidarité contre-révolutionnaire transnationale ${ }^{60}$. Ma thèse de doctorat a en effet eu pour objectif de montrer combien la menée de la guerre civile par les carlistes avait dépendu de multiples dimensions internationales, que l'on peut rappeler brièvement. D'abord, les carlistes s'appuyèrent tout au long du conflit sur des réseaux d'appui en Europe et aux Amériques qui leur fournirent argent, fournitures, armes et hommes, et qui constituèrent donc un véritable “arrière " pour une armée carliste qui n'en disposait pas vraiment en Espagne. La solidarité des contre-révolutionnaires du monde atlantique, et en particulier des légitimistes français, joua donc un rôle majeur dans la capacité même des carlistes à mener leur combat. En particulier, les opérations de financement permises par la mobilisation des catholiques de toute l'Europe et des riches familles légitimistes, ainsi que les circuits de contrebande d'armes qui acheminèrent fusils, munitions et canons parfois depuis fort loin furent décisifs ${ }^{61}$. Plus largement, la guerre de 1872-1876 vit se développer les traditionnelles manifestations de l'internationalisme politique, de la mobilisation par l'écrit au volontariat transnational, prouvant par là 
que la contre-révolution n'avait rien à envier, sur ce plan, à des cultures politiques plus avancées.

Ensuite, les carlistes excellèrent à mettre en relation ces réseaux de soutien transnationaux, principalement formés par des nobles, des ecclésiastiques et des exilés carlistes, avec les appuis locaux dont ils bénéficiaient dans les sociétés de frontière de la région pyrénéenne. Ce soutien transfrontalier à la cause de don Carlos procura aux carlistes une véritable zone de refuge dans les départements du sud de la France, d'où partaient aussi contrebandiers et passeurs qui acheminaient hommes et matériel de l'autre côté de la frontière au nez et à la barbe des douaniers et des gendarmes. L'histoire transnationale de la guerre carliste fut en effet aussi l'histoire d'une lutte contre les tentatives des États espagnol et français pour reprendre le contrôle de cette frontière et pour lutter de concert contre l'insurrection. En cela, l'internationale blanche, comme l'a nommée Jordi Canal, fit de la guerre carliste un enjeu de la diplomatie internationale de l'époque et non un simple conflit civil entre deux camps limités à l'Espagne. Il reste sans doute beaucoup à faire quant à la compréhension de ce soutien des populations de la frontière au carlisme, qui renvoie aussi aux raisons du soutien populaire à la contre-révolution.

Je distinguerais pour finir deux grands chantiers qui me semblent encore largement ouverts s'agissant de cette guerre carliste de 1872-1876. Le premier a trait au fonctionnement et à l'organisation de ce que l'on a pu appeler le proto-État ou le pseudo-État carliste. Dans les régions basques conquises en 1873, et qui formaient un territoire certes limité mais cohérent, les dirigeants carlistes construisirent en effet un embryon d'État qui aspirait à mettre en œuvre le programme politique de la contrerévolution espagnole ${ }^{62}$. Malgré la situation de guerre et la précarité du contrôle sur leur territoire, les carlistes mirent en place un semblant d'administration, et un début de législation. Si cet "État " a été étudié il y a quelques années par Julio Montero ${ }^{63}$, cette étude s'est limitée à une approche théorique et juridique de l'œuvre des carlistes, et des études par en bas, s'appuyant sur les réalisations concrètes, pourrait donner de la chair à cette réalité. En particulier, des travaux s'inspirant de l'histoire matérielle pourraient tirer un grand profit des collections des musées évoqués en introduction.

Enfin, il faudrait revenir sur la guerre elle-même et sur son déroulement depuis une approche inspirée de l'histoire sociale et de l'anthropologie. Trois chantiers mériteraient ainsi d'être creusés. D'abord, on connaît encore mal la sociologie et les motivations de ceux qui s'engagèrent dans l'armée de don Carlos. On sait que cet engagement ne reposa pas exclusivement sur le volontariat ${ }^{64}$, on connaît aussi globalement la composition des différents corps de l'armée carliste, mais on ne dispose que de connaissances sommaires sur les raisons qui poussèrent les uns et les autres à prendre les armes. Un deuxième chantier a trait aux pratiques de violence et à l'anthropologie du combat, qui a connu des renouvellements historiographiques importants ${ }^{65}$. Il y aurait énormément à faire de ce point de vue: la question des “ atrocités de guerre » et ses enjeux médiatiques et politiques ; la question sanitaire et humanitaire dans le conflit; les rapports, au sein du carlisme en guerre, entre armée constituée et bandes rassemblées sous la houlette de chefs charismatiques ; la pratique de la guérilla d'un point de vue stratégique et anthropologique etc.

Enfin, la place des civils dans le camp carliste est encore méconnue. On le sait, les formes de guerre s'inspirant de la guérilla supposent de pouvoir s'appuyer sur un tissu social qui soutient les combattants de diverses manières. À ce titre, de futurs travaux 
devront se pencher sur le rôle tenu par les femmes dans la guerre carliste, et plus généralement dans cette culture politique qui leur laissa une place paradoxale au regard des stéréotypes de genre qu'elle portait ${ }^{66}$. J'ajouterai en forme de point d'interrogation final, qu'il conviendra aussi de se pencher sur le curieux acharnement dont firent preuve les carlistes contre les voies de chemin de fer et les chefs de gare, ce qui renvoie à des considérations militaires stratégiques mais aussi à des réflexions sur les rapports entretenus par la contre-révolution avec les innovations scientifiques et techniques, manière de conclure cette réflexion qui visait à souligner combien le Sexennat fut un moment décisif dans l'évolution du carlisme, mais aussi symptomatique de la complexité des liens entre contre-révolution et modernité au XIX ${ }^{\mathrm{e}}$ siècle.

\section{NOTES}

1. Melchor FERRER, Historia del tradicionalismo español, Madrid, Editorial Católica Española, $1941-1979$, t. 23/2, p. 185

2. Id., t. $23 / 1$.

3. Antonio PIRALA, Historia contemporánea. Anales desde 1843 hasta la conclusión de la actual guerra civil, Madrid, Impr. de Manuel Tello, 1875, t. IV.

4. Jordi CANAL, El carlismo, Madrid, Alianza Editorial, 2000, chapitre 3.

5. Biblioteca de la Real Academia de la Historia (Madrid), fondo Antonio Pirala.

6. Archivo Histórico Nacional (Madrid), archivo de la familia de Borbón-Parma.

7. Guilhem CHAUVET, « Défendre la "cause" d'un siècle à l'autre : Maria de las Nieves de Braganza y de Borbón, trajectoire(s) légitimiste(s) d'une princesse exilée (1852-1941) », sous la direction de Jean-Philippe Luis, Université Clermont-Auvergne.

8. Archivo General de la Universidad de Navarra (Pampelune), fondo Melchor Ferrer.

9. Le mot, qu'on ne retrouve pas dans les dictionnaires contemporains, est attesté à l'époque du Sexennat chez Littré, qui précise qu'il s'agit d'un néologisme (Émile LITTRÉ, Dictionnaire de la langue française, Paris, L. Hachette, 1873-1874, t. 3, p. 1306). Bien qu'il n'ait visiblement pas prospéré, nous maintiendrons ici son usage, faute de mieux.

10. Pedro RÚJULA, «El antiliberalismo reaccionario » dans María Cruz ROMEO MATEO et María SIERRA ALONSo (coord.), La España liberal, 1833-1874, Zaragoza, Prensas de la Universidad de Zaragoza, 2014, p. 377-401.

11. À ce titre, il convient de souligner l'important retournement idéologique opéré par le carlisme à partir des années 1840 par rapport au canon politique des Bourbons du XVIII ${ }^{\mathrm{e}}$ siècle. La défense par les responsables carlistes des fameux fueros, qui garantissaient encore au XIX ${ }^{\mathrm{e}}$ siècle des droits particuliers aux provinces basques, tranchait avec le programme centralisateur mis en place à partir de Philippe $\mathrm{V}$ par les ascendants de don Carlos.

12. María Teresa DE BRAGANZA Y DE BORBóN, Mi carta a los Españoles, Paris, impr. de Cosson, 1865.

13. Jean-Clément MARTIN (dir), La contre-Révolution en Europe. XVIII -XIX ${ }^{e}$ siècles. Réalités politiques et sociales, résonances culturelles et idéologiques, Rennes, Presses Universitaires de Rennes, 2001 ; Jordi CANAL, « Repensar la historia de la contrarrevolución en la Europa del siglo XIX » dans El Carlismo 
en su tiempo: geografías de la contrarrevolución, Actas de las I Jornadas de estudio del carlismo 18-21 septiembre 2007, Estella, Pamplona, Gobierno de Navarra, 2008, p. 19-24.

14. Theo ARONSON, Venganza real. La corona de España, 1829-1968, Barcelona, Grijalbo, 1968, p. 118.

15. Pour le cas français, voir Alexandre DUPONT, «Le légitimisme, parent pauvre de l'historiographie? », Revue Historique, 2014/4, n 672, p. 887-909.

16. Simon SARLIN, Le légitimisme en armes : histoire d'une mobilisation internationale contre l'unité italienne, Rome, Presses de l'École Française de Rome, 2013.

17. Sur le cas français, voir Arthur HÉRISSON, «Les catholiques français face à l'unification italienne (1856-1871). Une mobilisation internationale de masse entre politique et religion », thèse d'histoire, Paris-1, 2018.

18. Jesús PABÓN. España y la cuestión romana, Madrid, Moneda y Crédito, 1972 ; Begoña URIGÜEN, Orígenes y evolución de la derecha española: el neo-catolicismo, Madrid, Consejo Superior de Investigaciones Científicas, 1986.

19. Agustín PACHECO FERNÁNDEZ et Francisco Javier SUÁREZ DE VEGA, Wils y el batallón de Zuavos Carlistas, Valladolid, Editorial Galland Books, 2019.

20. María Cruz ROMEO MATEO, "¿Sujeto católico femenino? Política y religión en España, 1854-1868 », Ayer, no 106, 2017, p. 79-104.

21. Jordi CANAL, El carlismo... op. cit., p. 120.

22. Vicente de manterola, Don Carlos o el petróleo, Madrid, impr. de Antonio Perez Dubrull, 1871.

23. Voir notamment Alexandre DUPONT, "Les carlistes au temps du Sexenio Democrático (1868-1874). Entre expérience parlementaire et tradition du soulèvement », Parlement(s), Revue d'histoire politique, HS 12, 2017, p. 63-82 ; Alexandre DUPONT, «Una politización paradójica. Carlismo, democracia e implicación popular durante el Sexenio Democrático », Investigaciones Históricas, época moderna y contemporánea, nº 37, 2017, p. 40-68.

24. Francisco Javier RAMÓN SOLANS et Pedro RÚJula (dir.), El desafío de la Revolución. Reaccionarios, antiliberales y contrarrevolucionarios (siglo XVIII y XIX), Granada, Comares, 2017.

25. Philippe BOUTRY et Michel CINQUIN, Deux pèlerinages au XIX siècle : Ars et Paray-le-Monial, Paris, Beauchesne, 1980 ; Francisco Javier RAmón solans, La Virgen del Pilar dice... Usos políticos nacionales de un culto mariano en la España contemporánea, Zaragoza, Prensas de la Universidad de Zaragoza, 2014.

26. Jordi CANAL, El carlisme català dins l'Espanya de la Restauració. Un assaig de modernització política (1888-1900), Vic, Eumo Editorial, 1998.

27. Begoña URIGÜEN, Orígenes y evolución..., op. cit.

28. Alexandre DUPONT, "The political structures of the carlism in exile. Politics from abroad, international solidarities and politics in the making", dans Catherine BRICE (ed.), Exile and the circulation of political practices, Cambridge, Cambridge Scholars, à paraître.

29. Biblioteca de la Real Academia de la Historia, Fondo Pirala, legajo 6863, « Actas originales de juntas o sesiones del Consejo presidido por D. Carlos empezando por la del 10 de Abril en París y concluyendo con la del 7 de Junio del mismo año 69 ».

30. Le sociologue Stéphane Dufoix a forgé ce concept dans les années 2000 pour désigner l'action politique des exilés, lorsque celle-ci visait à reprendre le pouvoir dans leur pays d'origine. Voir Stéphane Duforx, Politiques d'exil : Hongrois, Polonais et Tchécoslovaques en France après 1945, Paris, Presses universitaires de France, 2002.

31. Jacques LAGROYE, La politisation, Paris, Belin, 2003.

32. Esperanza CARPIZO BERGARECHE, La Esperanza carlista (1844-1874), San Sebastián de los Reyes, Actas, 2008.

33. Juan PARDO et Juantxo EGAÑA, Historia fotográfica de la última guerra carlista, Donostia, Txertoa, 2007. 
34. Sur cette politisation catholique, Gregorio DE LA FUENTE MONGE, «El enfrentamiento entre clericales y revolucionarios en torno a 1869 », Ayer, no 44, 2001, p. 127-150; Mikel URQUIJO GOITIA, Liberales y carlistas. Revolución y Fueros Vascos en el preludio de la última guerra carlista, Bilbao, Servicio Editorial, Universidad del País Vasco, 1994. Voir aussi, pour le cas français, la thèse d'Arthur Hérisson citée supra et Austin GoUGH, Paris et Rome : les catholiques français et le pape au XIXe siècle, Paris, Éditions de l'Atelier, Éditions Ouvrières, 1996.

35. Enriqueta SESMERO CUTANDA, Notables locales y carlismo en Bizkaia (ca. 1868-1876), Bilbao, Agirilan, 2003.

36. Pedro RÚjULA, Rebeldía campesina y primer carlismo: los orígenes de la guerra civil en Aragón (1833-1835), Zaragoza, Departamento de Educación y de Cultura, 1995 ; ID., Contrarrevolución, realismo y carlismo en Aragón y el Maestrazgo : 1820-1840, Zaragoza, Prensas universitarias de Zaragoza, 1998.

37. Pedro Rújula, «La guerra como aprendizaje político. De la guerra de la Independencia a las guerras carlistas » dans El Carlismo en su tiempo: geografías de la contrarrevolución, Actas de las I Jornadas de estudio del carlismo 18-21 septiembre 2007, Estella, Pamplona, Gobierno de Navarra, 2008, p. 41-63.

38. Mikel URQUIJO GOITIA, Liberales y carlistas..., op. cit.

39. Lluis Ferran TOLEDANO GONZÁLEZ, Entre el sermó i el trabuc : el carlisme català contra la revolució setembrina (1868-1872), Lleida, Pagès, 2001 ; Lluis Ferrán TOLEDANO GONZÁLEZ, Carlins i catalanisme : la defensa dels furs i de la religió a la darrera carlinada, 1868-1875, Sant Vicenç de Castellet, Farell, 2002.

40. Enriqueta SESMERO CUTANDA, Clases populares y carlismo en Bizkaia, 1850-1872, Bilbao, Universidad de Deusto, 2000.

41. Voir toutefois Maria Cruz MINA APAT, Fueros y revolucion liberal en Navarra, Madrid, Alianza Editorial, 1981 ; Juan PAN-MONTOJo, Carlistas y liberales en Navarra, (1833-1839), Pamplona, Gobierno de Navarra, 1989 ; Juan PAN-MONTOJo, «La revolución liberal y las transformaciones de la agricultura española », Areas: Revista internacional de ciencias sociales, ํㅡ 37, 2018, p. 29-43.

42. Voir le classique et très important : Ernest LABRouSSE, «1848, 1830, 1789. Comment naissent les révolutions? ", Actes du Congrès historique du centenaire de la révolution de 1848, Paris, PUF, 1949, p. 1-30.

43. Voir à ce titre le travail fondamental et pionnier de Álvaro PARÍS MARTíN, "Se susurra en los barrios bajos " : policía, opinión y política popular en Madrid, 1825-1827, thèse dirigée par Santos Madrazo Madrazo et Fernando Andrés Robles, Universidad Autónoma de Madrid, 2015.

44. Francisco TOMÁs Y VALIENTE, El marco político de la desamortización en España, Barcelona, Ariel, 1972.

45. Voir notamment Alexandre DUPONT, «Carlismo transfronterizo en los Pirineos en el siglo XIX. Unas paradojas a propósito del realismo popular ", Ayer, à paraître.

46. Giovanni LEVI, Le pouvoir au village. Histoire d'un exorciste dans le Piémont du XVII siècle, Paris, Gallimard, 1989. Il me semble que les travaux sur la période moderne proposés dans les années 1970 par Giovanni Levi, Carlo Ginzburg ou Natalie Zemon Davis peuvent encore constituer des sources d'inspiration épistémologique et méthodologique particulièrement fécondes.

47. Roger DUPUY et François LEBRUN (dir.), Les résistances à la Révolution: actes du colloque de Rennes, 17-21 septembre 1985, Paris, Imago, 1987.

48. Enriqueta SESMERO CUTANDA, Clases populares y carlismo en Bizkaia..., op. cit.

49. Voir par exemple Emilio de ARJONA, Pages d'histoire du parti carliste, Charles VII et D. Ramon Cabrera, par D. Emilio de Arjona. Traduit de l'espagnol, Paris, Féchoz, 1875.

50. Violencias fratricidas : carlistas y liberales en el siglo XIX. Actas de las II Jornadas de estudio del carlismo, 24-26 septiembre 2008, Estella, Pamplona, Gobierno de Navarra, 2009.

51. Norbert ELIAS, La dynamique de l'Occident, Paris, Calmann-Lévy, 1975 [1969] ; Jürgen HABERMAS, De l'éthique de la discussion, Paris, Flammarion, 2013 [1992]. 
52. Voir toutefois les importants travaux sur le sujet d'Eduardo GONZÁLEZ CALLEJA, par exemple, La razón de la fuerza. Orden público, subversión y violencia política en la España de la Restauración (1874-1917), Madrid, CSIC, 1998.

53. Gerald BRENAN, Le Labyrinthe espagnol : origines sociales et politiques de la guerre civile, Paris, Éditions Champ libre, 1984 [1943] ; Juan Pablo fusi et Jordi PALAFox, España 1808-1996. El desafío de la modernidad, Madrid, Espasa-Calpe, 1997.

54. Jordi CANAL, "Guerres civiles en Europe au XIX siècle, guerre civile européenne et Internationale blanche » dans Jean-Paul zÚÑIGA (dir.), Pratiques du transnational. Terrains, preuves, limites, Paris, Centre de Recherches Historiques, 2011, p. 57-77.

55. Outre les références déjà citées, voir Vincent GARMENDIA, La Segunda Guerra carlista (1872-1876), Madrid, Siglo XXI, 1976; Alexandre DUPONT, "Seconde guerre carliste», dans Jean-Clément MARTIN (dir.), Dictionnaire de la contre-révolution, Paris, Perrin, 2011.

56. Paul RICOEUR, «Remarques d'un philosophe », dans INSTITUT D'HISTOIRE DU TEMPS PRÉSENT, Écrire l'histoire du temps présent. En hommage à François Bédarida, Paris, CNRS Éditions, 1995.

57. Alexandre DUPONT, « Les carlistes au temps du Sexenio Democrático... », art. cit.

58. Sur cet épisode, je me permets de renvoyer à Alexandre DUPONT, «Une Internationale blanche. Les légitimistes français au secours des carlistes (1868-1883) », thèse sous la direction de Philippe Boutry et Pedro Rújula, Université Paris-1 et Universidad de Zaragoza, 2015, chapitre 11.

59. Alexandre DUPONT, «Entre exil et emprisonnement, l'originale expérience des carlistes en France (1868-1876)» dans Nicolas BEAUPRÉ et Karine RANCE (dir.), Arrachés et déplacés. Réfugiés politiques, prisonniers de guerre et déportés (1789-1918), Clermont-Ferrand, Presses de l'Université Blaise Pascal, 2016, p. 145-164 ; Alexandre DUPONT, «

60. Alexandre DUPONT, « Une Internationale blanche... », vol. cit.

61. Alexandre DUPONT, «France et Espagne face à la contrebande d'armes pro-carliste (1872-1876) : entre crise diplomatique et impuissance militaire », Stratégique, no 118/1, 2018, p. 193-204.

62. Vincent GARMENDIA, La Segunda Guerra carlista..., op. cit.

63. Julio MONTERo, El Estado Carlista. Principios teóricos y práctica política (1872-1876), Madrid, Aportes XIX, 1992.

64. José Ramón URQUIJO GOITIA, «¿Voluntarios o quintos? Reclutamiento y deserción en la Primera Guerra Carlista ", dans Violencias fratricidas : carlistas y liberales en el siglo XIX. Actas de las II Jornadas de estudio del carlismo, 24-26 septiembre 2008, Estella, Pamplona, Gobierno de Navarra, 2009, p. 99-186.

65. Voir par exemple Stéphane AUDOIN-ROUZEAU, Combattre. Une anthropologie historique de la guerre moderne (XIX ${ }^{e}-\mathrm{XXI}^{e}$ siècle), Paris, Seuil, 2008.

66. Quelques pistes: Alexandre DUPONT, «Soignantes et consolatrices. Femmes contrerévolutionnaires pendant la Seconde guerre carliste (1872-1876)", Genre et Histoire, 2017/2 (http://genrehistoire.revues.org/2695); ID., "Le genre de la contre-révolution au xIxe siècle ", Encyclopédie pour une histoire nouvelle de l'Europe [en ligne], 2016, mis en ligne le 29/01/2018, consulté le 02/09/2018. Permalien : ehne.fr/node/1201 


\section{RÉSUMÉS}

Cet article propose un bilan historiographique des trente dernières années de recherches portant sur le carlisme espagnol pendant le Sexennat Démocratique (1868-1874). À partir d'une discussion du concept de modernité, il propose d'interpréter ce moment de l'histoire de la contre-révolution espagnole comme un processus de modernisation paradoxale de cette culture politique. L'article s'arrête sur trois aspects représentatifs de cette modernisation: la reconstruction du carlisme et sa structuration en une organisation qui préfigure par ses pratiques les partis politiques modernes; la politisation des classes populaires carlistes et la place de ces processus de politisation subalternes dans l'évolution idéologique et tactique de cette organisation; le recours à la guerre et ses implications en termes de violence politique et de ressources stratégiques à plusieurs échelles. L'article propose également de nouvelles pistes de réflexion et de travail sur cette famille politique essentielle dans l'histoire de l'Espagne au XIX ${ }^{\mathrm{e}}$ siècle.

Este artículo propone un balance historiográfico de los últimos treinta años de investigación sobre el carlismo español durante el Sexenio Democrático (1868-1874). A partir de una discusión del concepto de modernidad, propone interpretar este momento en la historia de la contrarrevolución española como un proceso de modernización paradójica de esta cultura política. El artículo se detiene en tres aspectos representativos de esta modernización: la reconstrucción del carlismo y su estructuración en una organización que prefigura los partidos políticos modernos; la politización de las clases populares carlistas y el papel de estos procesos de politización subalternos en la evolución ideológica y táctica de esta organización ; la elección de la guerra y sus implicaciones en términos de violencia política y de recursos estratégicos en varias escalas. El artículo propone también nuevas pistas de reflexión y de trabajo sobre esta familia política esencial en la historia de España en el siglo XIX.

This article proposes an historiographic review of the last 30 years' researches on Spanish carlism during the Six Democratic Years (1868-1874). From a discussion of the concept of modernity, it proposes to interprete this moment of the history of the Spanish counterrevolution as a process of paradoxical modernization of this political culture. The article stresses on three aspects representative of this modernization: the reconstruction of carlism and its structuration in an organization that prefigures the modern political parties; the politicization of the carlist popular classes and the part of these subaltern processes of politicization in the ideological and tactical evolution of this organization; the choice of the war and its implications in terms of political violence and strategic resources at various scales. The article also proposes new paths for reflection and research on this important political family of 19th century Spain.

\section{INDEX}

Mots-clés : Carlisme, modernisation, politisation, mobilisation politique, Seconde guerre carliste, historiographie.

Palabras claves : Carlismo, modernización, politización, movilización política, Segunda guerra carlista, historiografía.

Keywords : Carlism, modernization, politicization, political mobilization, Second carlist war, historiography. 
AUTEUR

ALEXANDRE DUPONT

Université Strasbourg 